\title{
Early Fetal Echocardiography: Ready for Prime Time?
}

\author{
Fadi G. Mirza, M.D. ${ }^{1,2}$, Samuel T. Bauer, M.D. ${ }^{2}$, Ismee A. Williams, M.D., M.S. ${ }^{3}$, and Lynn L. \\ Simpson, M.D. ${ }^{2}$ \\ ${ }^{1}$ Department of Obstetrics and Gynecology, American University of Beirut Medical Center, Beirut, \\ Lebanon \\ ${ }^{2}$ Division of Maternal-Fetal Medicine, Department of Obstetrics and Gynecology, Columbia \\ University Medical Center, New York, New York \\ ${ }^{3}$ Division of Pediatric Cardiology, Department of Pediatrics, Columbia University Medical Center, \\ New York, New York
}

\begin{abstract}
The objective of this study was to examine the performance of early fetal echocardiography as a screening tool for major cardiac defects in a high-risk population. Fetal echocardiograms performed at 12 to 16 weeks were reviewed. Cases that did not undergo a follow-up echocardiogram at 18 to 22 weeks were excluded. Results of the early and follow-up echocardiograms were compared. Over a 4-year period, 119 early fetal echocardiograms were recorded. Of those, $81(68 \%)$ had follow-up fetal echocardiograms. Results of the early echocardiogram were normal in 77 of $81(95.1 \%)$ cases. Of these, the follow-up was normal in 75 of these 77 cases; in the remaining 2 , the follow-up raised suspicion for a ventricular septal defect (VSD) in one and persistent left superior vena cava in the other. On the other hand, the early echocardiogram was abnormal in 4 (4.9\%) cases: (1) atrioventricular canal defect, with the followup demonstrating a VSD; (2) hypoplastic right ventricle and transposition of the great arteries, confirmed on follow-up; (3) VSD and coarctation of the aorta, confirmed on follow-up. In the fourth case, the early echocardiogram suspected a VSD and right-left disproportion, yet the follow-up was normal. In conclusion, early fetal echocardiography appears to be a reasonable screening tool for major cardiac defects.
\end{abstract}

\section{Keywords}

fetal echocardiography; fetal heart; congenital heart disease; prenatal diagnosis

Congenital heart disease is considered the most common birth defect, impacting 3 to 8 per 1000 live births. It is estimated that $\sim 1 \%$ of fetuses will show sonographic evidence of a cardiac anomaly on a screening ultrasound performed during the second trimester of pregnancy. ${ }^{1-3}$ Congenital heart disease can have a major impact on pregnancy outcomes. Intrauterine demise complicates 20 to $30 \%$ of fetuses with identified cardiac defects, and for certain lesions, neonatal death occurs in 40 to $60 \%$ of cases, with long-term survival rates ranging from 15 to $40 \%{ }^{4-8}$ Timely prenatal diagnosis of cardiac anomalies can offer families the opportunity to receive appropriate counseling regarding the anticipated fetal and

\footnotetext{
Copyright $(92012$ by Thieme Medical Publishers, Inc.

Address for correspondence and reprint requests Fadi G. Mirza, M.D., American University of Beirut, Department of Obstetrics and Gnecology, 3 Dag Hammarskjold Plaza, New York, NY 10017 (fmirza@ aub.edu.lb).

The preliminary results of this study were presented as an abstract at the World Congress on Ultrasound in Obstetrics and Gynecology held in Hamburg, Germany in September 2009.
} 
neonatal outcomes. It may also allow for informed decisions regarding continuation of pregnancy. Moreover, prenatal diagnosis allows for transfer of care to a facility with expert neonatology, pediatric cardiology, and pediatric cardiac surgery services, a resource that can have a major impact on the outcome of affected neonates. ${ }^{9,10}$

The majority of fetal cardiac anomalies occurs in low-risk populations; hence, prenatal ultrasound represents an important primary screening modality. ${ }^{11}$ A detailed fetal echocardiogram is, however, indicated in high-risk patients, with the intent to examine the fetal heart in greater detail than that obtained at the time of a screening ultrasound. ${ }^{12-15} \mathrm{At}$ our institution, which serves as a tertiary care referral center, fetal echocardiography is generally used to screen major cardiac defects in the following cases: abnormal-appearing heart on prenatal ultrasound; fetal tachycardia, bradycardia, or persistent irregular rhythm on clinical or screening ultrasound examination; maternal/family risk factors for cardiovascular disease such as a parent, sibling, or first-degree relative with congenital heart disease; pregestational diabetes mellitus; systemic lupus erythematosus; teratogen exposure during a vulnerable period; performance of transplacental therapy; and enlarged nuchal translucency (NT) and cystic hygroma on first-trimester risk assessment for aneuploidy. It appears that the latter is gaining popularity, and, of interest, an association has been reported in numerous studies between increased NT and congenital heart disease even in the chromosomally normal fetus. ${ }^{16-24}$ The risk of congenital heart disease rises with increasing NT, and referral for fetal echocardiography is generally warranted if the NT is greater than $2.5 \mathrm{MoM}$, the 99th percentile, or 3.0 to $3.5 \mathrm{~mm}$. As for cystic hygroma, the risk of congenital anomalies has been reported to be as high as $30 \%$, with more than $70 \%$ of those being cardiac. ${ }^{25}$

Development of the human cardiovascular system occurs by 3 to 6 weeks following conception. ${ }^{12}$ By 11 weeks of gestation, the position of the fetal heart and the spatial relations of the great arteries are similar to those in later gestation. Hence, although fetal echocardiography is typically performed in the second trimester, improved resolution of ultrasound has allowed for fetal echocardiography to be performed earlier in gestation. It is imperative though that the as low as reasonably achievable principle be observed when adjusting controls that affect the acoustic output and by considering transducer dwell times. Numerous studies have demonstrated the feasibility of first or early second trimester cardiac imaging. ${ }^{26-29}$ The accuracy of early fetal echocardiography in the diagnosis of congenital heart disease has been encouraging as well. ${ }^{30-37}$ The objective of our study was to examine the performance of early fetal echocardiography in a high-risk population at a single tertiary care referral center in the United States.

\section{Material and Methods}

This was a retrospective study, approved by the Institutional Review Board, of all early fetal echocardiograms performed at our center between 12 0/7 and 15 6/7 weeks of gestation. In these studies, two-dimensional ultrasound, M-mode echocardiography, and color and pulsed Doppler velocimetry were used to evaluate and document the following, whenever possible: cardiac position, situs, and axis; four-chamber view; five-chamber view; three-vessel view; short-axis view of the right ventricular outflow tract; long-axis view left ventricular outflow tract; aortic arch; ductal arch; superior vena cava; inferior vena cava; pulmonary veins; ductus venosus; direction of foramen ovale flow; atrioventricular valves; semilunar valves; fetal heart motion, rate, and rhythm; and cardiac qualitative systolic function. The early fetal echocardiogram was performed by a maternal-fetal medicine specialist using a transabdominal approach, although transvaginal sonography supplemented suboptimal transabdominal views, when needed. All patients who underwent an early fetal echocardiogram were informed of the results and also of the need for a follow-up (standard) fetal echocardiogram by a pediatric cardiologist at approximately 18 to 22 weeks of 
gestation. Yet, several patients did not return to our center for this purpose and assumingly obtained a follow-up study at their referring institutions. The cases that did not undergo a follow-up examination at our center were excluded from this analysis. The results of the early and follow-up fetal echocardiograms were then compared in the included cases.

\section{Results}

Between March 2006 and March 2010, 119 early fetal echocardiograms were performed. Of those, 81 had follow-up fetal echocardiograms at our center and were included in this study. Ten of these $81(12.3 \%)$ early fetal echocardiograms included a transvaginal examination due to suboptimal transabdominal visualization. Demographic information and indications for the early fetal echocardiogram are presented in Tables 1 and 2, respectively. As illustrated in Table 3, results of the early fetal echocardiograms were normal in 77 (95.1\%) of the 81 cases. Of these, the follow-up fetal echocardiogram was normal in 75 cases. In one of these cases reported as normal on the early study, the follow-up fetal echocardiogram raised suspicion for a peri-membranous ventricular septal defect (VSD). In the second case, persistent left superior vena cava to the coronary sinus was noted, without evidence of pulmonary venous obstruction, anomalous pulmonary venous return or cor triatriatum. The early fetal echocardiogram, in turn, was abnormal in 4 of the 81 cases (4.9\%): (1) suspected atrioventricular canal defect, with the follow-up fetal echocardiogram demonstrating a perimembranous VSD; (2) suspected hypoplastic right ventricle and transposition of the great arteries, which was confirmed on follow-up; and (3) suspected VSD and a left ventricular outflow tract that appeared smaller than the right ventricular outflow tract raising suspicion for coarctation of the aorta, which was confirmed on follow-up. In the fourth case, the early fetal echocardiogram raised the suspicion for a VSD and noted a right-left side disproportion, yet the follow-up fetal echocardiogram was normal. As such, the sensitivity of the early echocardiogram compared with the standard study was calculated as $60.0 \%$ (95\% confidence interval $[\mathrm{CI}], 23.0-88.0$ ), although the specificity was $98.7 \%$ (95\% CI, 93.0-99.8). The positive predictive value of this study was $75.0 \%$ (95\% CI, 30.1-95.4), although the negative predictive value reached $97.4 \%$ (95\% CI, 91.0-99.3).

\section{Discussion}

Congenital heart disease is the most common form of birth defects. Population screening studies have shown that this condition affects $\sim 5 / 1000$ to $8 / 1000$ newborns, with approximately one-half causing major disease. ${ }^{12}$ While there are no clear definitions of "major" and "minor" disease, the former category generally refers to congenital heart disease that is clearly evident at or shortly after birth. Examples include cyanotic heart disease, severe valvular stenosis, and large VSD. Minor disease, in turn, includes small defects such as smaller VSD and mild valvular stenosis. Specialized neonatal care is often indicated for infants with suspected congenital heart disease. Therapeutic interventions, such as prompt infusion of prostaglandin E1 or balloon atrial septostomy for defects that require postnatal maintenance of fetal flow pathways, can improve neonatal survival when congenital heart disease is detected prenatally and the timing, mode, and location of delivery is planned. Immediate institution of extracorporeal membrane oxygenation may also impact the outcome of cases complicated by certain complex cardiac defects.

Prenatal diagnosis of congenital heart disease is, hence, pivotal, thus the role of prenatal ultrasound as a primary screening modality in low-risk patients. In turn, a more detailed cardiac evaluation with a fetal echocardiogram is indicated in the setting of recognized risk factors for congenital heart disease. ${ }^{12-15}$ If a fetal echocardiogram is indicated, it is typically performed during the second trimester. Yet, improved resolution of ultrasound has enabled fetal echocardiography to be performed earlier in gestation. As might be expected, there has 
been a growing interest in this approach, which may potentially provide earlier reassurance to high-risk patients; alternatively, identification of congenital heart disease at an early gestational age may allow for more optimal counseling and additional management options in certain circumstances. Numerous studies have examined and demonstrated the feasibility of first or early second-trimester cardiac imaging. ${ }^{26-29}$ Success rates were consistently above $90 \%$ for visualizing the four-chamber and proximal outflow tracts in major referral centers with less consistent visualization of the distal outflow arches and venous return, and the ideal timing appeared to be the 13th to 14 th week of gestation. ${ }^{12}$ Transvaginal sonography is often a component of early fetal echocardiography. In fact, in the obese patient, transvaginal sonography in the late first trimester may be superior to the midtrimester examination as it avoids insonation through the abdominal pannus.

The accuracy of early fetal echocardiography in the diagnosis of congenital heart disease has also been encouraging. Becker and Wegner prospectively evaluated 3094 fetuses between 11 0/7 and 13 6/7 weeks over a 7-year period at a single center in Germany. ${ }^{30}$ This cohort included women with singleton and multiple gestations opting for early anomaly screening. The latter included a fetal echocardiogram in addition to the fetal anatomical survey. Detection of major congenital heart disease by early fetal echocardiography reached $84 \%$. Undetected abnormalities included absent pulmonary valve, atrioventricular septal defect, transposition of the great arteries, tetralogy of Fallot, and total anomalous pulmonary venous drainage. Three of 6 undetected anomalies were not identified prenatally, although two patients did not present for a follow-up echocardiogram in the mid-trimester. In the second largest series, also from Germany, Smrcek et al retrospectively reviewed all singleton pregnancies that underwent a fetal echocardiogram between 11 0/7 and 13 6/7 weeks for which outcome data were available. ${ }^{31}$ In contrast to the former study, the cohort in this study included 2165 high-risk patients, specifically referred for a fetal echocardiography. About $63 \%$ of pregnancies affected by congenital heart disease were identified at the time of the early echocardiogram, with an additional $24 \%$ detected prior to delivery. Abnormalities detected included atrioventricular canal defects, tetralogy of Fallot, truncus arteriosus, tricuspid valve dysplasia, pulmonary atresia or stenosis, aortic coarctation, and VSD. Other smaller series have also examined the performance of early echocardiography ${ }^{32-37}$ Again the majority of cardiac defects present were identified by early fetal echocardiography, with sensitivity ranging from 58 to $92 \%$. Arch abnormalities and minor defects such as septal defects were the most difficult to identify. A more recent systemic review examined the accuracy of ultrasound screening for congenital heart disease in the first trimester on the basis of 10 studies. ${ }^{38}$ Most studies in this review included high-risk patients with a previous affected child or increased NT. The pooled sensitivity of all studies was $85 \%$, while the specificity reached $99 \%$.

Our study examined the performance of early fetal echocardiography in a high-risk population at a major referral center in the United States. This study included a significant portion of patients with increased NT and cystic hygroma as the indication for early fetal echocardiography. This is not unexpected given the increased popularity and access to firsttrimester risk assessment, leading to the identification of a new subset of pregnancies at risk for congenital heart disease earlier in pregnancy. Our contemporary data support earlier reports regarding the accuracy of early fetal echocardiography for the detection of congenital heart disease. A limitation of this study was its retrospective nature, although all examinations were performed by one of two providers and followed an official protocol that outlined the study indications and components. Another limitation of this study was the inability to evaluate the performance of the early fetal echocardiogram in comparison to a neonatal study, as some infants did not undergo neonatal evaluation while others delivered at their referring institutions and the results of the neonatal study, if performed, were not available for our review. A unique strength, on the other hand, was that while a 
perinatologist performed the early fetal echocardiogram, a pediatric cardiologist always performed the follow-up study. Another limitation for this study is the relatively small number of cases included, which is partially attributed to the relatively new trend of early fetal echocardiography in the United States. As such, larger, prospective studies are required.

In conclusion, fetal echocardiography remains the mainstay for the diagnosis of congenital heart disease in utero, and it appears to be feasible and accurate when performed during the first and early second trimesters. Although this study further supports early fetal echocardiography as a reasonable screening tool for major cardiac defects, early fetal echocardiography should be viewed as an adjunct, rather than replacement, to standard second-trimester fetal echocardiography. Furthermore, counseling regarding antenatal and postnatal outcome and management should also take into consideration that even when major cardiac defects are identified, the precise diagnosis and the full spectrum of defects are often not made until later in gestation. Finally, early fetal echocardiography should generally be reserved for patients at risk for congenital heart disease and should be performed in experienced centers.

\section{Acknowledgments}

We would like to acknowledge the statistical assistance of William Strohsnitter, D.Sc.

\section{References}

1. Hoffman JI, Kaplan S. The incidence of congenital heart disease. J Am Coll Cardiol. 2002; 39(12): 1890-1900. [PubMed: 12084585]

2. Montaña E, Khoury MJ, Cragan JD, Sharma S, Dhar P, Fyfe D. Trends and outcomes after prenatal diagnosis of congenital cardiac malformations by fetal echocardiography in a well defined birth population, Atlanta, Georgia, 1990-1994. J Am Coll Cardiol. 1996; 28(7):1805-1809. [PubMed: 8962570]

3. Buskens E, Steyerberg EW, Hess J, Wladimiroff JW, Grobbee DE. Routine prenatal screening for congenital heart disease: what can be expected? A decision-analytic approach. Am J Public Health. 1997; 87(6):962-967. [PubMed: 9224177]

4. Crawford DC, Chita SK, Allan LD. Prenatal detection of congenital heart disease: factors affecting obstetric management and survival. Am J Obstet Gynecol. 1988; 159(2):352-356. [PubMed: 3407692]

5. Sharland GK, Lockhart SM, Chita SK, Allan LD. Factors influencing the outcome of congenital heart disease detected prenatally. Arch Dis Child. 1991; 66(3):284-287. [PubMed: 1827247]

6. Vergani P, Mariani S, Ghidini A, et al. Screening for congenital heart disease with the four-chamber view of the fetal heart. Am J Obstet Gynecol. 1992; 167(4 Pt 1):1000-1003. [PubMed: 1415383]

7. Davis GK, Farquhar CM, Allan LD, Crawford DC, Chapman MG. Structural cardiac abnormalities in the fetus: reliability of prenatal diagnosis and outcome. Br J Obstet Gynaecol. 1990; 97(1):27-31. [PubMed: 2407282]

8. Smythe JF, Copel JA, Kleinman CS. Outcome of prenatally detected cardiac malformations. Am J Cardiol. 1992; 69(17):1471-1474. [PubMed: 1590238]

9. Bonnet D, Coltri A, Butera G, et al. Detection of transposition of the great arteries in fetuses reduces neonatal morbidity and mortality. Circulation. 1999; 99(7):916-918. [PubMed: 10027815]

10. Chang AC, Huhta JC, Yoon GY, et al. Diagnosis, transport, and outcome in fetuses with left ventricular outflow tract obstruction. J Thorac Cardiovasc Surg. 1991; 102(6):841-848. [PubMed: 1960988]

11. Sharland G. Routine fetal cardiac screening: what are we doing and what should we do? Prenat Diagn. 2004; 24(13):1123-1129. [PubMed: 15614849]

12. Johnson B, Simpson LL. Screening for congenital heart disease: a move toward earlier echocardiography. Am J Perinatol. 2007; 24 (8):449-456. [PubMed: 17992711] 
13. Cheitlin MD, Alpert JS, Armstrong WF, et al. ACC/AHA Guidelines for the clinical application of echocardiography. Circulation. 1997; 95(6):1686-1744. [PubMed: 9118558]

14. Allan LD. Fetal echocardiography. Clin Obstet Gynecol. 1988; 31(1):61-79. [PubMed: 3359728]

15. Small M, Copel JA. Indications for fetal echocardiography. Pediatr Cardiol. 2004; 25(3):210-222. [PubMed: 15360114]

16. Hyett J, Moscoso G, Papapanagiotou G, Perdu M, Nicolaides KH. Abnormalities of the heart and great arteries in chromosomally normal fetuses with increased nuchal translucency thickness at 11-13 weeks of gestation. Ultrasound Obstet Gynecol. 1996; 7 (4):245-250. [PubMed: 8726875]

17. Hyett JA, Perdu M, Sharland GK, Snijders RS, Nicolaides KH. Increased nuchal translucency at 10-14 weeks of gestation as a marker for major cardiac defects. Ultrasound Obstet Gynecol. 1997; 10(4):242-246. [PubMed: 9383874]

18. Mavrides E, Cobian-Sanchez F, Tekay A, et al. Limitations of using first-trimester nuchal translucency measurement in routine screening for major congenital heart defects. Ultrasound Obstet Gynecol. 2001; 17(2):106-110. [PubMed: 11251916]

19. Zosmer N, Souter VL, Chan CSY, Huggon IC, Nicolaides KH. Early diagnosis of major cardiac defects in chromosomally normal fetuses with increased nuchal translucency. Br J Obstet Gynaecol. 1999; 106(8):829-833. [PubMed: 10453834]

20. Schwärzler P, Carvalho JS, Senat MV, Masroor T, Campbell S, Ville Y. Screening for fetal aneuploidies and fetal cardiac abnormalities by nuchal translucency thickness measurement at 10 14 weeks of gestation as part of routine antenatal care in an unselected population. Br J Obstet Gynaecol. 1999; 106(10):1029-1034. [PubMed: 10519427]

21. Michailidis GD, Economides DL. Nuchal translucency measurement and pregnancy outcome in karyotypically normal fetuses. Ultrasound Obstet Gynecol. 2001; 17(2):102-105. [PubMed: 11251915]

22. Simpson LL, Malone FD, Bianchi DW, et al. Nuchal translucency and the risk of congenital heart disease. Obstet Gynecol. 2007; 109 (2 Pt 1):376-383. [PubMed: 17267839]

23. Bahado-Singh RO, Wapner R, Thom E, et al. First Trimester Maternal Serum Biochemistry and Fetal Nuchal Translucency Screening Study Group. Elevated first-trimester nuchal translucency increases the risk of congenital heart defects. Am J Obstet Gynecol. 2005; 192(5):1357-1361. [PubMed: 15902108]

24. Vogel M, Sharland GK, McElhinney DB, et al. Prevalence of increased nuchal translucency in fetuses with congenital cardiac disease and a normal karyotype. Cardiol Young. 2009; 19(5):441445. [PubMed: 19638249]

25. Malone FD, Ball RH, Nyberg DA, et al. FASTER Trial Research Consortium. First-trimester septated cystic hygroma: prevalence, natural history, and pediatric outcome. Obstet Gynecol. 2005; 106 (2):288-294. [PubMed: 16055577]

26. Haak MC, Twisk JWR, Van Vugt JMG. How successful is fetal echocardiographic examination in the first trimester of pregnancy? Ultrasound Obstet Gynecol. 2002; 20(1):9-13. [PubMed: 12100411]

27. Souka AP, Pilalis A, Kavalakis Y, Kosmas Y, Antsaklis P, Antsaklis A. Assessment of fetal anatomy at the 11-14-week ultrasound examination. Ultrasound Obstet Gynecol. 2004; 24(7):730734. [PubMed: 15586371]

28. Vimpelli T, Huhtala H, Acharya G. Fetal echocardiography during routine first-trimester screening: a feasibility study in an unselected population. Prenat Diagn. $2006 ; 26(5): 475-482$. [PubMed: 16652404]

29. Smrcek JM, Berg C, Geipel A, Fimmers R, Diedrich K, Gembruch U. Early fetal echocardiography: heart biometry and visualization of cardiac structures between 10 and 15 weeks' gestation. J Ultrasound Med. 2006; 25(2):173-182. quiz 183-185. [PubMed: 16439780]

30. Becker R, Wegner RD. Detailed screening for fetal anomalies and cardiac defects at the 11-13week scan. Ultrasound Obstet Gynecol. 2006; 27(6):613-618. [PubMed: 16570262]

31. Smrcek JM, Berg C, Geipel A, et al. Detection rate of early fetal echocardiography and in utero development of congenital heart defects. J Ultrasound Med. 2006; 25(2):187-196. [PubMed: 16439781] 
32. Carvalho JS, Moscoso G, Tekay A, Campbell S, Thilaganathan B, Shinebourne EA. Clinical impact of first and early second trimester fetal echocardiography on high risk pregnancies. Heart. 2004; 90 (8):921-926. [PubMed: 15253970]

33. Comas Gabriel C, Galindo A, Martínez JM, et al. Early prenatal diagnosis of major cardiac anomalies in a high-risk population. Prenat Diagn. 2002; 22(7):586-593. [PubMed: 12124694]

34. Gembruch U, Knöpfle G, Bald R, Hansmann M. Early diagnosis of fetal congenital heart disease by transvaginal echocardiography. Ultrasound Obstet Gynecol. 1993; 3(5):310-317. [PubMed: 12797253]

35. Huggon IC, Ghi T, Cook AC, Zosmer N, Allan LD, Nicolaides KH. Fetal cardiac abnormalities identified prior to 14 weeks' gestation. Ultrasound Obstet Gynecol. 2002; 20(1):22-29. [PubMed: 12100413]

36. McAuliffe FM, Trines J, Nield LE, Chitayat D, Jaeggi E, Hornberger LK. Early fetal echocardiography — a reliable prenatal diagnosis tool. Am J Obstet Gynecol. 2005; 193(3 Pt 2): 1253-1259. [PubMed: 16157147]

37. Weiner Z, Lorber A, Shalev E. Diagnosis of congenital cardiac defects between 11 and 14 weeks' gestation in high-risk patients. J Ultrasound Med. 2002; 21(1):23-29. [PubMed: 11794398]

38. Rasiah SV, Publicover M, Ewer AK, Khan KS, Kilby MD, Zamora J. A systematic review of the accuracy of first-trimester ultrasound examination for detecting major congenital heart disease. Ultrasound Obstet Gynecol. 2006; 28(1):110-116. [PubMed: 16795132] 
Table 1

Demographic Information

\begin{tabular}{|l|l|}
\hline & Mean \\
\hline Age (y) & 33.2 \\
\hline Gravidity & 2.7 \\
\hline Parity & 0.8 \\
\hline Plurality & 1.3 \\
\hline GA at time of early fetal echo (wk) & 14.7 \\
\hline GA at time of standard fetal echo (wk) & 20.9 \\
\hline
\end{tabular}

echo, echocardiogram; GA, gestational age; wk, weeks; y, years. 
Table 2

Indications for Early Fetal Echocardiogram

\begin{tabular}{|l|l|}
\hline & Number of Patients (\%) \\
\hline Family or personal history of CHD & $27(33)$ \\
\hline Pregestational DM & $22(27)$ \\
\hline Cystic hygroma and increased NT & $20(25)$ \\
\hline Multiple gestations with MC placentation & $11(13)$ \\
\hline Abnormal fetal karyotype & $5(6)$ \\
\hline
\end{tabular}

CHD, congenital heart disease; DM, diabetes mellitus; MC, monochorionic; NT, nuchal translucency. 
Table 3

Results

\begin{tabular}{|l|l|}
\hline Results of Early Fetal Echocardiogram & Results of Standard Fetal Echocardiogram \\
\hline Normal $(n=77)$ & Normal $(n=75)$ \\
& Abnormal $(n=2)$ \\
VSD & Persistent left superior vena cava \\
\hline Abnormal $(n=4)$ & Normal $(n=1)$ \\
& Abnormal $(n=3)$ \\
& VSD \\
& TGA \\
& VSD and coarctation of the aorta \\
\hline
\end{tabular}

TGA, transposition of the great arteries; VSD, ventricular septal defect. 